\title{
Plasma Waves in Anisotropic Superconducting Films Below and Above the Plasma Frequency
}

\author{
Mauro M. Doria ${ }^{a}$, Gilberto Hollauer $^{b}$, F. Parage ${ }^{c}$, and O. Buisson ${ }^{c}$ \\ ${ }^{a}$ Instituto de Física, Universidade Federal Fluminense, C.P. 100.093 \\ Niterói 24001-970 RJ, Brazil. \\ ${ }^{b}$ Departamento de Física, Pontifícia Universidade Católica do Rio de Janeiro, Rio de Janeiro \\ 22452-970 RJ, Brazil. \\ ${ }^{c}$ Centre de Recherches sur les Très Basses Températures, Laboratoire Associé à l'Université \\ Joseph Fourier, C.N.R.S., BP 166, 38042 Grenoble-Cédex 9, France.
}

\begin{abstract}
We consider wave propagation inside an anisotropic superconducting film sandwiched between two semi-infinite non-conducting bounding dieletric media such that along the c-axis, perpendicular to the surfaces, there is a plasma frequency $\omega_{p}$ below the superconducting gap. Propagation is assumed to be parallel to the surfaces in the dielectric medium, where amplitudes decay exponentially. Below $\omega_{p}$, the amplitude also evanesces inside the film, and we retrieve the experimentally measured lower dispersion relation branch, $\omega \propto \sqrt{\beta}$, and the recently proposed higher frequency branch, $\omega \propto 1 / \sqrt{\beta}$. Above $\omega_{p}$, propagation is of the guided wave type, i.e., a dispersive plane wave confined inside the film that reflects into the dielectric interfaces, and the modes are approximately described by $\omega \approx \omega_{p} \sqrt{1+\left(\beta / \beta_{0}\right)^{2}}$, where $\beta_{0}$ is discussed here.
\end{abstract}




\section{INTRODUCTION}

The experimental measurement of a plasma edge in the infrared reflectivity of HighTc bulk superconductorst目 $\mathrm{La}_{2-x} \mathrm{Sr}_{x} \mathrm{CuO}_{4}, \mathrm{YBa}_{2} \mathrm{Cu}_{3} \mathrm{O}_{8-x}$ 目, and $\mathrm{Bi}_{2} \mathrm{Sr}_{2} \mathrm{CaCu}_{2} \mathrm{O}_{8}$ 目目 has brought a renewed interest in collective oscillations with the Cooper pair density. Previously, such studies were hampered by the well-known argument 9 that the Coulomb interaction shifts the frequency of such oscilations to above the gap frequency. Recent theoretical studies 10 - 14 support the view of a plasma oscillations along the c-axis, the direction orthogonal to the $\mathrm{CuO}_{2}$ planes, caused by a large superconducting gap and a high anisotropy in these materials.

In superconducting films, plasma oscillations have distinct properties. They exist in films regardless of their anisotropy or layered structure, and regardless of the type of pairing or even of the critical temperature value. This mode was predicted $15,12,16$ some time ago and was bothly observed in thin granular aluminium films, in the hundreds of $M H z$ range 18 , and in thin $\mathrm{YBa}_{2} \mathrm{Cu}_{3} \mathrm{O}_{8-x}$ films 19 , in the higher frequency range of hundreds of $\mathrm{GHz}$.

The study of propagating modes in films is not a new research field, they have been measured since long ago, in several materials ranging from metals 2022 to semiconductors 23 , and also discussed in the theoretical literature 25,24.26. The novelty for the superconducting film is the very low frequency where these modes are observed and the strong temperature dependence, explained by a collective oscillation with the Cooper pair density. Recently27 it has been proposed that, similarly to metals and semiconductors, two branches of propagating modes should be observed in highly anisotropic superconducting films, e.g., the High-Tc compounds. The experimental observation of these two modes can provide a method to independently measure the London penetration lengths, parallel and perpendicular to the film surface.

In this paper we include into the study of mode propagation in films the existence of a c-axis plasma frequency, $\omega_{p}$, inside the superconducting state. We only consider the c-axis perpendicular to the surfaces since this configuration is the most easily grown nowadays. 
The propagation of plasma modes above and below $\omega_{p}$, their dispersion relations are our goals in the present paper. We restrict our study to waves that propagate along the surfaces of the superconducting film and evanesce in the dielectric medium perpendicularly to the surfaces. Two very distinct behaviors are predicted, namely, field amplitudes that also evanesce inside the film away from the interfaces $\left(\omega<\omega_{p}\right)$, and confined propagation, i.e., waves propagate inside the superconductor along an oblique direction and reflect at the dielectric-superconductor interfaces $\left(\omega>\omega_{p}\right)^{29}$. In many aspects this last case resembles propagation in optic fibers. In conclusion this paper considers a plasma frequency $\omega_{p}$ inside the superconducting state and studies its effects into wave propagation in films.

The study of plasma modes in superconducting films with a plasma frequency $\omega_{p}$ has been previously considered by Artemenko and Kobel'kov ${ }^{28}$ in the context of kinetic equations for Green functions generalized to the case of layered superconductors with weak interlayer coupling. The present paper provides a more complete study of such modes than theirs because it shows that there are symmetric and antisymmetric modes for $\omega<\omega_{p}$ and discusses their several distinct regimes. The antisymmetric mode is intimately connected to the transverse current component, thus being highly sensitive to the transverse London penetration length, in the same fashion that the symmetric mode depends on the longitudinal London penetration length. We claim that these properties can be used to gain a better understanding of the transverse and longitudinal supercurrent components in anisotropic and layered films. Besides, the present study is done in a framework distinct from those authors, we take the simplest possible theory, the London-Maxwell theory for anisotropic materials, supposedly valid when all lengths are larger than the inter-layer separation $a$. In the context of this theory we determine exactly the mode frequency, $\omega$, as a function of its wavelength along the surfaces, $\beta$, and of the remaining parameters: the two London penetration lengths, transverse $\left(\lambda_{\perp}\right)$ and longitudinal $\left(\lambda_{\|}\right)$to the surfaces; the dielectric constant of the non-conducting medium exterior to the film, $\tilde{\varepsilon}$; the film thickness $d$; and the isotropy and frequency independent $\varepsilon_{s}$, the simplest phenomenological choice for the superconductor's static dieletric constant. 
This paper is organized as follows. In the next section, ([I]), we derive the dispersion relation equations, above and below the plasma frequency, using the London-Maxwell theory, and solve them exactly. Our study is restricted to identical top and bottom dielectric media. Similar conclusions should also apply to the most general asymmetric case. Section III deals with the $\omega<\omega_{p}$ case, where we study the two possible branches, the so-called symmetric (lower) and antisymmetric (upper) branches, and their three possible regimes: optical, coupled, and asymptotic. For each branch we derive, from our exact solution, useful approximated expressions for each of the above regimes. In this fashion many of the previous film studies are shown to be suitable approximations of our exact solutions. In section IV we study wave propagation above $\omega_{p}$ and, obtain, from our exact dispersion relation, the several dispersion relation branches found by Artemenko and Kobel'kov28. We show that symmetry still plays an important role and the two branches below $\omega_{p}$ split into several ones, the symmetry of the state being determined by the number of half-wavelengths that fit perpendicularly to the film. In section $\nabla$ we apply the present theory to the High-Tc

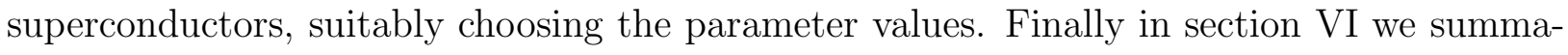
rize our major results. The proof of some of results from our exact solution, such as the approximated expressions, are the subject of appendix $\nabla 11$.

\section{THE LONDON-MAXWELL THEORY APPLIED TO ANISOTROPIC SUPERCONDUCTING FILMS}

In this section we apply the London-Maxwell theory to describe wave propagation in a superconducting film sandwiched between two identical non-conducting dielectric media 30 . An external electromagnetic wave of frequency $\omega$, and wavenumber $k \equiv \omega / c$, is absorbed by the superconducting film, producing a mode whose dispersion relation is $\omega(\beta)$. We choose a coordinate system where the two plane parallel surfaces separating the superconducting film to the dielectric medium are at $x=d / 2$ and $x=-d / 2$. Propagation takes place along the $z$ axis such that all fields can be expressed as $F_{i}(x) \exp [-i(\beta z-\omega t)]$. At this point 
we introduce the time dependence $\exp (i \omega t)$ to all fields into the basic equations governing the system. Current transport inside the film is described by the first London equation,

$$
i \omega \mu_{0} \lambda_{\|}^{2} \mathbf{J}_{\|}=\mathbf{E}_{\|}, \quad i \omega \mu_{0} \lambda_{\perp}^{2} J_{\perp}=E_{\perp}
$$

where $\mathbf{E}_{\|}$and $\mathbf{E}_{\perp}$ are the field components parallel and perpendicular to the film surfaces, respectively. The electromagnetic coupling, given by Maxwell's equations,

$$
\begin{aligned}
\nabla \cdot \mathbf{D} & =0 \\
\nabla \cdot \mathbf{H} & =0 \\
\nabla \times \mathbf{E} & =-i \omega \mu_{0} \mathbf{H} \\
\nabla \times \mathbf{H} & =i \omega \mathbf{D} \quad \text { where } \quad \mathbf{D}=\epsilon_{s} \mathbf{E}-i \mathbf{J} / \omega
\end{aligned}
$$

shows that the superconductor dielectric constant is tensorial, $\mathbf{D}=\epsilon_{0} \varepsilon \cdot \mathbf{E}$.

$$
\varepsilon=\left(\begin{array}{ccc}
\varepsilon_{\perp} & 0 & 0 \\
0 & \varepsilon_{\|} & 0 \\
0 & 0 & \varepsilon_{\|}
\end{array}\right) \quad \varepsilon_{\perp}=\varepsilon_{s}-\frac{1}{\left(k \lambda_{\perp}\right)^{2}} \quad \varepsilon_{\|}=\varepsilon_{s}-\frac{1}{\left(k \lambda_{\|}\right)^{2}}
$$

Phenomenological theories, such as the present, describe the superconductor only at energies much lower than the pair breaking threshold. The frequency $\omega$ is much smaller than the frequency defined by the superconducting gap. Consequently the frequency is much smaller than the plasma frequency along the $C u O_{2}$ planes, $k \lambda_{\|} \ll 1$, thus rendering a negative, and large in modulus, dielectric tensor component parallel to the surfaces.

$$
\left|\varepsilon_{\|}\right| \gg 1 \quad \varepsilon_{\|} \approx-\frac{1}{\left(k \lambda_{\|}\right)^{2}}
$$

In the present model the c-axis plasma frequency is reached when the dielectric tensor component perpendicular to the surfaces becomes null.

$$
\varepsilon_{\perp}\left(\omega=\omega_{p}\right)=0 \quad \omega_{p}=\frac{c}{\sqrt{\varepsilon_{s}} \lambda_{\perp}}
$$

Hence $\varepsilon_{\perp}$ changes sign as $\omega$ crosses $\omega_{p}$. 
Solving Maxwell's equations for this particular geometry gives two independent sets of field components, $\left(H_{x}, E_{y}, H_{z}\right)$, the transverse electric $(\mathrm{TE})$, and $\left(E_{x}, H_{y}, E_{z}\right)$, the transverse magnetic (TM) propagating modes. For the TM mode the interfaces superconductordielectric acquire superficial charge densities. Curiously anisotropy also implies in a volumetric charge density inside the film, $\rho=-i \nabla \cdot \mathbf{J} / \omega=\epsilon_{0}\left(1-\varepsilon_{\perp} / \varepsilon_{\|}\right) \partial E_{\perp} / \partial x$, although it is not responsible for these low frequency propagating modes. In fact such modes were first observed in nearly isotropic thin granular aluminum films 18 . In conclusion the TM mode supports low frequency travelling waves because of the oscillating superficial charge densities that couple the non-conducting dielectric medium to the superconducting film, their ratio given by,

$$
\frac{\sigma(x=d / 2)}{\sigma(x=-d / 2)}=-\frac{\partial E_{z} /\left.\partial x\right|_{x=d / 2}}{\partial E_{z} /\left.\partial x\right|_{x=-d / 2}}
$$

The TM field equations are given below, for the dielectric medium, $(x \geq d / 2$ and $x \leq-d / 2)$,

$$
E_{x}=i \frac{\beta}{\tilde{\tau}^{2}} \frac{\partial E_{z}}{\partial x}, \quad H_{y}=i \epsilon_{0} \frac{\omega \tilde{\varepsilon}}{\tilde{\tau}^{2}} \frac{\partial E_{z}}{\partial x}, \quad \frac{\partial^{2} E_{z}}{\partial x^{2}}-\tilde{\tau}^{2} E_{z}=0, \quad \tilde{\tau}^{2}=\beta^{2}-k^{2} \tilde{\varepsilon}
$$

and for the superconducting strip $(-d / 2 \leq x \leq d / 2)$,

$$
E_{x}=i \frac{\beta \varepsilon_{\|}}{\tau^{2} \varepsilon_{\perp}} \frac{\partial E_{z}}{\partial x}, \quad H_{y}=i \epsilon_{0} \frac{\omega \varepsilon_{\|}}{\tau^{2}} \frac{\partial E_{z}}{\partial x}, \quad \frac{\partial^{2} E_{z}}{\partial x^{2}}-\tau^{2} E_{z}=0 \quad \tau^{2}=\frac{\varepsilon_{\|}}{\varepsilon_{\perp}} \beta^{2}-k^{2} \varepsilon_{\|}
$$

In this paper we seek propagating modes that evanesce in the dielectric medium, namely display exponential decay in the dielectric. This condition is $\tilde{\tau}^{2}=\beta^{2}\left[1-(\omega / \beta v)^{2}\right]>0$, and can be interpreted as demanding a phase velocity, $\omega / \beta$, smaller than the speed of light in the dielectric, $v \equiv c / \sqrt{\tilde{\varepsilon}}$. Hence above the film $(x \geq d / 2)$ one gets that,

$$
E_{z}=\tilde{E}_{o} \exp (-\tilde{\tau} x)
$$

From the other side exponential decay inside the film is not sure to occur because the sign of $\tau^{2}$ is not uniquely defined. This sign determines different physical regimes, and for this reason, we introduce the below and above plasma condition and study their respective $\tau^{2}$ regions. Hereafter, in order to simplify further discussions, we will choose the nonconducting dielectric medium to have the largest static constant, $\tilde{\varepsilon}>\varepsilon_{s}$. 
$\underline{\omega<\omega_{p}}$ In this case both $\varepsilon_{\|}$and $\varepsilon_{\perp}$ have the same sign. Introducing the Eq.(7) approximation, one gets that $\tau^{2} \approx\left|\varepsilon_{\|} / \varepsilon_{\perp}\right| \beta^{2}+1 / \lambda_{\|}^{2}>0$. The planes of constant phase are $z=$ const. and field amplitudes also evanesce inside the superconductor.

$$
E_{z}=E_{o} \exp (-\tau x)+F_{o} \exp (\tau x), \quad \tau^{2}>0
$$

$\underline{\omega>\omega_{p}}$ In this case $\varepsilon_{\|}<0$ and $\varepsilon_{\perp}>0$. Even within the Eq.(17) approximation, $\tau^{2} \approx-\left|\varepsilon_{\|} / \varepsilon_{\perp}\right| \beta^{2}+1 / \lambda_{\|}^{2}$ has no definite sign. The mode may be evanescent or propagative inside the superconductor. However the evanescent modes disappear for $\tilde{\varepsilon}>\varepsilon_{s}$ and then one gets that $\tau^{2}<0$. In this case the planes of constant phase are $\beta z \pm \tau^{\prime} x=$ const. $\left(\tau^{\prime 2}=-\tau^{2}>0\right)$, and we fall into the case of confined propagation, with an oblique incidence well defined at the interfaces.

$$
E_{z}=E_{o} \exp \left(-i \tau^{\prime} x\right)+F_{o} \exp \left(i \tau^{\prime} x\right), \quad \tau^{\prime 2}=-\frac{\varepsilon_{\|}}{\varepsilon_{\perp}} \beta^{2}+k^{2} \varepsilon_{\|}>0
$$

In summary for the present purposes the sign of $\tau^{2}$ is uniquely determined below and above $\omega_{p}$ by the sign of the ratio $\varepsilon_{\|} / \varepsilon_{\perp}$.

The dispersion relations follow from the continuity of the ratio $H_{y} / E_{z}$ at a single interface, say $x=d / 2$, once assumed that the longitudinal field $E_{z}$ is either an even or an odd function with respect to the $x=0$ plane. This is possible because the two dielectric media, above and below the film, are equal.

Table I : The four possible dispersion relations and $E_{z}$.

$$
\begin{array}{|c|c|c|}
E_{z} & \text { Dispersion Relation } \\
\hline E_{o z} & \cosh (\tau x) & \frac{\tau \tilde{\varepsilon}}{\tilde{\tau} \varepsilon_{\|}}=-\tanh \left(\tau \frac{d}{2}\right) \\
E_{o z} & \sinh (\tau x) & \frac{\tau \tilde{\varepsilon}}{\tilde{\tau} \varepsilon_{\|}}=-\frac{1}{\tanh \left(\tau \frac{d}{2}\right)} \\
E_{o z} & \cos \left(\tau^{\prime} x\right) & \frac{\tau^{\prime} \tilde{\varepsilon}}{\tilde{\tau} \varepsilon_{\|}}=-\tan \left(\tau^{\prime} \frac{d}{2}\right) \\
E_{o z} & \sin \left(\tau^{\prime} x\right) & \frac{\tau^{\prime} \tilde{\varepsilon}}{\tilde{\tau} \varepsilon_{\|}}=\frac{1}{\tan \left(\tau^{\prime} \frac{d}{2}\right)}
\end{array}
$$

At this point we introduce some dimensionless variables useful in the study of the above dispersion relations. 
Table II : Dimensionless Variables.

\begin{tabular}{|c|c|c|c|c|} 
Retardation & Anisotropy & Dielectric & Thickness & Wave Number \\
\hline$\gamma \equiv \frac{\omega / \beta}{v}$ & $R \equiv\left(\frac{\lambda_{\perp}}{\lambda_{\|}}\right)^{2}$ & $r \equiv \frac{\tilde{\varepsilon}}{\varepsilon_{s}}$ & $A \equiv\left(\frac{\lambda_{\|}}{d}\right)^{2}$ & $X=\left(\beta \lambda_{\|}\right)^{2}$
\end{tabular}

We cast some of our previous results in the dimensionless variables formalism. The condition of evanescence along the direction orthogonal to the surfaces is $0 \leq \gamma \leq 1$, since according to Eq.(12), one has that $E_{z}=\tilde{E}_{o} \exp \left(-\sqrt{\frac{X}{A}} \sqrt{1-\gamma^{2}} x / d\right)$. The dominance of the incident wavelength over London's penetration along the surface, which gave Eq.(7), becomes $\gamma^{2} \ll$ $r / X$. The dielectric component ratio, within the Eq.(17) approximation, is $\varepsilon_{\perp} / \varepsilon_{\|} \approx(1 / R)(1-$ $\left.R \gamma^{2} X / r\right)$. Thus the $\omega<\omega_{p}$ and $\omega>\omega_{p}$ regimes correspond to $R \gamma^{2} X / r<1$ and $R \gamma^{2} X / r>$ 1, respectively. The c-axis (High-Tc compounds) is perpendicular to the surfaces and so $R \geq 1$

In the following, we obtain the exact solution for each of the Eq.(15) dispersion relations considering the dimensionless variables $d \tau\left(\omega<\omega_{p}\right)$ and $d \tau^{\prime}\left(\omega>\omega_{p}\right)$ as a parameter $s$, whose range is still to be determined below and above $\omega_{p}$. Therefore we seek the dimensionless curve, $\gamma(X)$, in parametric form, $[X(s), \gamma(s)]$, and immediately obtain the dispersion relation, $[\beta(s), \omega(s)]=\left[\sqrt{X(s)} / \lambda_{\|},\left(v / \lambda_{\|}\right) \gamma(s) \sqrt{X(s)}\right]$.

$\underline{\omega<\omega_{p}}$ We introduce the dimensionless parameter,

$$
t \equiv d \tau=\frac{1}{\sqrt{A}} \sqrt{\frac{X R}{1-\frac{\gamma^{2} X R}{r}}+1}
$$

Using the Eq.(16) dimensionless variables, the Eq.(15) dispersion relations become,

Table III : The dispersion relations for $\omega<\omega_{p}$

$$
\begin{array}{|cc|c|}
E_{z} & \text { Dispersion Relation } \\
\hline E_{o z} & \cosh (t x / d) & \sqrt{A X} \frac{\gamma^{2}}{\sqrt{1-\gamma^{2}}}=\frac{\tanh (t / 2)}{t} \\
E_{o z} & \sinh (t x / d) & \sqrt{A X} \frac{\gamma^{2}}{\sqrt{1-\gamma^{2}}}=\frac{1}{t \tanh (t / 2)}
\end{array}
$$


To find their solution, first obtain $X(t, \gamma)$ from Eq.(17), and then introduce it into Eq.(18). We obtain a second degree equation for $\gamma(t)^{2}$ with two roots: one negative the other positive. The negative root does not correspond to propagating modes and only the positive is left.

$$
\begin{aligned}
\gamma_{I}(t)^{2} & =\frac{\left(A t^{2}-1\right)-r+\sqrt{\left[\left(A t^{2}-1\right)+r\right]^{2}+4 \frac{A r^{2}}{R}\left(A t^{2}-1\right) g_{I}(t)}}{2\left(A t^{2}-1\right)\left[1+\frac{A r}{R} g_{I}(t)\right]} \\
X(t) & =\frac{r}{R} \frac{A t^{2}-1}{\gamma_{I}(t)^{2}\left(A t^{2}-1\right)+r}
\end{aligned}
$$

$I=\{S, A\}$ labels the $E_{z}$ symmetry: $g_{A}(t)=t^{2} \tanh ^{2}(t / 2)$ and $g_{S}(t)=t^{2} / \tanh ^{2}(t / 2)$ are associated to the antisymmetric and the symmetric modes, respectively. According to the above equations the parameter $t$ range is $[1 / \sqrt{A}, \infty]$.

$\underline{\omega>\omega_{p}}$ Through Eq.(14) we define a new dimensionless parameter.

$$
t^{\prime} \equiv d \tau^{\prime}=\frac{1}{\sqrt{A}} \sqrt{\frac{X R}{\frac{\gamma^{2} X R}{r}-1}-1}
$$

Introducing the Eq.(16) dimensionless variables into the Eq.(15) dispersion relations gives that,

Table IV : The dispersion relations $\omega>\omega_{p}$

$$
\begin{array}{|c|c|}
E_{z} & \text { Dispersion Relation } \\
\hline E_{o z} \cos \left(t^{\prime} x / d\right) & \sqrt{A X} \frac{\gamma^{2}}{\sqrt{1-\gamma^{2}}}=\frac{\tan \left(t^{\prime} / 2\right)}{t^{\prime}} \\
E_{o z} \sin \left(t^{\prime} x / d\right) & \sqrt{A X} \frac{\gamma^{2}}{\sqrt{1-\gamma^{2}}}=-\frac{1}{t^{\prime} \tan \left(t^{\prime} / 2\right)}
\end{array}
$$

To solve them, express Eq.(21) as $X\left(t^{\prime}, \gamma\right)$ and introduce it back into Eq.(22) obtaining a second degree equation for $\gamma\left(t^{\prime}\right)^{2}$, whose solution is,

$$
\begin{aligned}
\gamma_{I}\left(t^{\prime}\right)^{2} & =\frac{\left(A t^{\prime 2}+1\right)+r \pm \sqrt{\left[\left(A t^{\prime 2}+1\right)-r\right]^{2}-4 \frac{A r^{2}}{R}\left(A t^{\prime 2}+1\right) h_{I}\left(t^{\prime}\right)}}{2\left(A t^{\prime 2}+1\right)\left[1+\frac{A r}{R} h_{I}\left(t^{\prime}\right)\right]} \\
X_{I}\left(t^{\prime}\right) & =\frac{r}{R} \frac{A t^{\prime 2}+1}{\gamma_{I}\left(t^{\prime}\right)^{2}\left(A t^{\prime 2}+1\right)-r}
\end{aligned}
$$


Like in the previous case, $I=\{S, A\}$ gives the $E_{z}$ parity: $h_{A}\left(t^{\prime}\right)=t^{\prime 2} \tan ^{2}\left(t^{\prime} / 2\right)$ and $h_{S}\left(t^{\prime}\right)=t^{\prime 2} / \tan ^{2}\left(t^{\prime} / 2\right)$ for the antisymmetric and symmetric modes, respectively.

The discussion of the suitable parameter range is more involving in the present case. According to Eq.(22) the function $t^{\prime} \tan \left(t^{\prime} / 2\right)$ must remain negative for the antisymmetric problem whereas $\tan \left(t^{\prime} / 2\right) / t^{\prime}$ must be positive for the symmetric case. This restricts the parameter range $t^{\prime}$ to the intervals, $[2 N \pi,(2 N+1) \pi]$ for symmetric and $[(2 N+1) \pi, 2(N+$ 1) $\pi$ ] for antisymmetric, where $N$ is an integer larger or equal to zero.

Our exact parametrized solutions $\omega(\beta)$ are ready for applications, just requiring the numerical parameters. Before doing so in section $\mathrm{V}$, we find convenient to describe their physical properties in the next two sections.

\section{DISPERSION RELATION BELOW THE PLASMA FREQUENCY}

In this section we study the properties of propagating modes below $\omega_{p}$. We find convenient to summarize their major physical properties and introduce some approximated expressions, each describing a different regime of the exact $\omega<\omega_{p}$ dispersion curve. We leave to the appendix VII the proof that the previous section exact results do justify our picture and yield the approximated expressions. Below the plasma frequency the field amplitudes inside the film evanesce from the surfaces, and this exponential decay is characterized by $\tau$, according to Eq.(13). The choice of film thickness, $d$, is very important in order to assure sufficient coupling between the two surfaces. For extremely thick films the surfaces decouple and the symmetric and the antisymmetric modes are the same, only independent surface plasma modes exist in this situation. Indeed, in case of strong coupling between the two surfaces three distinct regimes are possible for both symmetric and antisymmetric modes. In sequence of increasing $\beta$ we call them optical, coupled and asymptotic (see Fig.(目)).

Close to the origin $\beta \approx 0$, the modes are optical, that is, they are essentially plane waves travelling in a dielectric medium. There is almost no exponential decay in the surrounding 
dielectric semi-spaces up to appreciable distances, $\tilde{\tau} \approx 0$, according to Eq.(12). Propagation occurs with the speed of light in the dielectric, $(\gamma \approx 1)$. The kinetic energy of the superconducting carriers is negligible compared to the magnetic energy of the mode. The superconducting film contributes very weakly to this regime. In the optical regime one has, approximately, the linear behavior $\omega \approx v \beta$.

Slowly the condensate's kinetic energy increases with $\beta$ until finally both energies become comparable and a first cross-over takes place. This is the onset of the coupled regime, a slow mode $(\gamma<1)$, where the condensate's kinetic energy dominates over the magnetic energy, the film and the dielectric are strongly coupled. This is the true plasma mode regime. Such evanescent propagating modes are known 15 17 27, here we discuss the effects of a c-axis plasma frequency $\omega_{p}$ below the gap 28 . In the coupled regime inside the film, the fields evanesce from the surfaces very smoothly resulting into two types of coupling between the superficial plasmons, which are the antisymmetric and the symmetric modes. For the symmetric mode its dispersion relation has been theoretically studied by many authorst 17 and experimentally observed 1819 in the past:

$$
\omega_{s} \approx \frac{v}{\lambda_{\|}} \sqrt{\frac{d \beta}{2}}
$$

Recently 27 it has been proposed that the symmetric mode is just the lowest frequency branch. In fact, there is an upper (antisymmetric) branch mode, that can also be experimentally observed for highly anisotropic High-Tc superconducting films:

$$
\omega_{a} \approx \frac{v}{\lambda_{\perp}} \sqrt{\frac{1}{d \beta / 2+\varepsilon_{s} / \tilde{\varepsilon}}}
$$

Because the antisymmetric branch is the highest in frequency, it is more sensitive to the effects of the plasma frequency $\omega_{p}$. For $d \beta / 2$ comparable (or smaller) to $\varepsilon_{s} / \tilde{\varepsilon}$, this branch becomes difficult to observe since $\omega_{a} \approx \omega_{p}$. For $d \beta / 2$ dominant over $\varepsilon_{s} / \tilde{\varepsilon}$, the symmetric and antisymmetric relations are proportional to $\sqrt{\beta}$ and $1 / \sqrt{\beta}$, respectively.

The cross-over frequency between the optical and the symmetric regime is obtained at the intersection of the two branches: 


$$
\omega_{\text {cross }, s}=\frac{v d}{2 \lambda_{\|}^{2}}
$$

The cross-over between optical and antisymmetric modes is implicitly given by the unique real solution:

$$
\left(\frac{d}{2 v}\right) \omega_{\text {cross }, a}^{3}+\left(\frac{\varepsilon_{s}}{\tilde{\varepsilon}}\right) \omega_{c r o s s, a}^{2}-\left(\frac{v}{\lambda_{\perp}}\right)^{2}=0
$$

For the antisymmetric mode, the optical and the coupled frequencies are, respectively, an increasing and a decreasing function of $\beta$. Thus the cross-over $\omega_{\text {cross }, a}$ also gives an estimate of the maximum frequency, associated to the peak seen in the antisymmetric curve (Fig.(2)).

By increasing $\beta$, evanescence inside the superconductor becomes stronger ( $\tau$ is large), up to the point where the surfaces are nearly decoupled. We have just reached the asymptotic regime. Thus for sufficiently high $\beta$, symmetric and antisymmetric modes converge to the same asymptotic frequency,

$$
\omega_{a s}=\omega_{p} \sqrt{\left(\frac{1+\sqrt{1+4\left(\frac{\tilde{\varepsilon} \lambda_{\|}}{\varepsilon_{s} \lambda_{\perp}}\right)^{2}}}{\left.1+2\left(\frac{\tilde{\varepsilon} \lambda_{\|}}{\varepsilon_{s} \lambda_{\perp}}\right)^{2}+\sqrt{1+4\left(\frac{\tilde{\varepsilon} \lambda_{\|}}{\varepsilon_{s} \lambda_{\perp}}\right.}\right)^{2}}\right)}
$$

Notice that it is always true that $\omega_{a s}<\omega_{p}$. It is easy to check in the following extreme cases of a very high dieletric constant $\left(\tilde{\varepsilon} / \varepsilon_{s} \gg \lambda_{\perp} / \lambda_{\|}\right)$, and of the opposite case, namely, when anisotropy plays a more important role than the dielectric constant, $\left(\tilde{\varepsilon} / \varepsilon_{s} \ll \lambda_{\perp} / \lambda_{\|}\right)$. In the former case we obtain27 $\omega_{a s}=v / \sqrt{\lambda_{\perp} \lambda_{\|}}$, and in the latter $\omega_{a s}=\omega_{p}$. In summary we have just reviewed the major features of the three possible regimes for both the symmetric and the antisymmetric dispersion relations.

We find that the dielectric constant ratio $r$ must be sufficiently high in order to prevent that the $\beta$ range be extremely limited for the coupled regime. In the next section ( $\mathbb{V}$ ) we study such crossovers in case of the High-Tc parameter values. The criterion for the disapperance of the coupled antisymmetric and symmetric regimes is just given by an asymptotic frequency $\omega_{\text {as }}$ smaller than $\omega_{\text {cross }, s}$ and $\omega_{\text {cross,a }}$, respectively. As an example consider the case of an extremely large anisotropy $\left(\lambda_{\perp} \rightarrow \infty\right)$, where we get that $\omega_{a s} \approx \omega_{p} \rightarrow 0$, eventually smaller 
than the crossover frequencies of Eq.(27) and Eq.(28). In this limit the dispersion relation goes almost directly from the optical to the asymptotic regime.

\section{DISPERSION RELATION ABOVE THE PLASMA FREQUENCY}

In this section propagating modes inside the superconducting film with the frequency larger than $\omega_{p}$ are considered. We summarize their major physical properties, and leave to the appendix VII their derivation from the exact parametrized dispersion relation. We are again interested in modes that evanesce in the dieletric medium. As discussed before, $\tau^{\prime}$ is always real with the experimental parameter choice of $\tilde{\varepsilon}>\varepsilon_{s}$. The propagation of light inside the film can be understood by a superposition of two dispersive plane waves, $\exp \left\{-i\left[ \pm \tau^{\prime}(\beta) x+\beta z-\omega(\beta) t\right]\right\}$, regarded as incident and reflected waves, thus with a well defined angle of incidence at the interfaces superconductor-dielectric. Thus while outside the film light is still evanescent, in its interior the propagation can be pictured through ray optics (see Fig.(11)), similarly to an optical fiber.

The exact parametrized plasma relation, given by Eq.(23) and Eq.(24), leads to the simple approximated dispersion relation, in the vicinity of $\omega / \beta \approx v$,

$$
\omega_{M}=\omega_{p} \sqrt{1+\frac{(\beta d)^{2}}{(\pi M)^{2}+\left(d / \lambda_{\|}\right)^{2}}\left(\frac{\lambda_{\perp}}{\lambda_{\|}}\right)^{2}}
$$

where $\mathrm{M}$ is a positive integer, odd for the symmetric modes $(M=2 N+1)$ and even for the antisymmetric modes $(M=2(N+1))$. This approximated expression was first obtained by Artemenko and Kobel'kov28. The $M^{t h}$ curve satisfies the evanescence condition, $\gamma \leq 1$, for $\beta>\beta_{\text {init }}(M)$.

$$
\beta_{\text {init }}(M)=\frac{1}{\lambda_{\perp} \sqrt{\frac{\varepsilon_{s}}{\tilde{\varepsilon}}-\frac{1}{(\pi M)^{2}}\left(\frac{d}{\lambda_{\|}}\right)^{2}}}
$$

At the particular value $\beta=\beta_{\text {init }}(M)$, the wave vector along the $\mathrm{x}$-direction is given by $\tau^{\prime} d=M \pi$ where $M$ is exactly the number of half-wavelength that fit perpendicularly to the film. For $\beta>\beta_{\text {init }}(M)$, the relation between $\tau^{\prime}$ and $M$ is not so simple because the wave 
is also in the dielectric medium. In this case $M$ just determines the number of $E_{z}$ extrema along the x-direction (see Fig.(11)). For instance, when just one half-wavelength fits into the film we are facing the $M=1$ symmetric mode, which has just a single $E_{z}$ extremum (maximum). We notice that the frequency of the plasma modes decreases for increasing M. For $\beta<\beta_{\text {init }}(M)$ we have that $\gamma>1$ and the modes are not evanescent, they are plane waves travelling in the dielectric medium. They have an oblique incidence at the surfaces and to see this just take the plane wave $\exp \{-i[\tilde{\tau} x+\beta z-\omega t]\}$ with the speed of light in the dielectric, $v=\omega / \sqrt{\tilde{\tau}^{2}+\beta^{2}}$. One obtains that $\tilde{\tau}=\beta \sqrt{\gamma^{2}-1}$, showing that we are in a $\gamma$ regime not studied here.

The present theory eventually breaks down because large $\tau^{\prime}$ means an infinitesimally small wavelength inside the film along the $x$ axis. For this reason a physical cut-off must be put into this theory, the inter-plane separation $a$. The upper limit $\tau_{\max }^{\prime}$ is determined when a x-direction half-wavelength fits into the interplane distance $a$.

$$
\tau_{\max }^{\prime}=\pi / a=M_{\max } \pi / d
$$

Introducing $M_{\max }$ in the approximated dispersion relation Eq.(30), gives the lowest frequency plasma mode: $\omega_{\text {min }}=\omega_{p} \sqrt{1+\left(\beta \lambda_{J} / \pi\right)^{2}}$, where $\lambda_{J} \equiv a \lambda_{\perp} / \lambda_{\|}$is the Josephson penetration depth.

Beyond $\tau_{\max }^{\prime}$ more elaborate models should provide a description of the condensate in such scale. This can render the theory's usage quite limited, in case the ratio $d / a$ is small, because for very few half-wavelengths inside the film we reach the cut-off limit. However the applicability of this model above $\omega_{p}$ is not restricted to a small ratio $d / a$ because the propagating mode has constant amplitude inside the film, and so, no matter how far apart the surfaces are, there will always be waves reflected at the surfaces. For this reason the major conclusions of this section, namely, the existence of slow modes above the plasma frequency, corresponding to dispersive plane waves inside the film, must remain valid even near the cut-off limit.

In the next section we take the standard parameter values for the high-Tc material and 
discuss the properties of such propagating modes below and above the plasma frequency.

\section{APPLICATIONS}

For our applications we choose the following set of parameters for the High-Tc ceramic superconductors. The static dielectric constant ${ }^{0}$ is $\varepsilon_{s} \approx 30$. The zero-temperature London penetration length along the $\mathrm{CuO}_{2}$ planes is $\lambda_{C O}=0.15 \mu \mathrm{m} 31$. While the anisotropy, $\lambda_{\perp} / \lambda_{\|}$, is 5 for $\mathrm{YBa}_{2} \mathrm{Cu}_{3} \mathrm{O}_{8-x}$ 31, it has been changing in the past for $\mathrm{Bi}_{2} \mathrm{Sr}_{2} \mathrm{CaCu}_{2} \mathrm{O}_{x}$, ranging from $10^{2}$, mainly from torque measurements studies31, to much higher values 33 . We show here that choosing the anisotropy between these two values can have important effects on the properties of the dispersion relations for $\omega<\omega_{p}$. There is also the compound $\mathrm{Tl}_{2} \mathrm{Ba}_{2} \mathrm{CaCuO}$ compound 32 with $\lambda_{\perp} / \lambda_{\|} \approx 90$.

Fig.(1) provides a pictorial intuitive view of wave propagation in the film below and above the plasma frequency. Below $\omega_{p}$ the instantaneous electric field, as well as the component $E_{z}$, are shown for both symmetric and antisymmetric fields. The surface charges are the sole sources of propagating electric fields. The superficial charge arrangement has strong consequences for the electric field distribuiton inside the film, leading to symmetric and antisymmetric modes, found at distinct frequency ranges, the latter being an upper mode. To understand the effects of anisotropy, or a layered structure, into these modes, where the $\mathrm{CuO}_{2}$ planes are parallel to the film surface, consider the transverse and longitudinal field and supercurrent components. $E_{x}$ and $J_{x}$ are very intense for the antisymmetric mode and nearly zero for the symmetric one, and $E_{z}$ and $J_{z}$, are the dominant components for the symmetric mode but not for the antisymmetric one. Thus the relevant penetration depths for the symmetric and the antisymmetric modes must be $\lambda_{\|}$and $\lambda_{\perp}$, respectively. The anisotropy $\lambda_{\perp}>\lambda_{\|}$hardens the system along the c-axis thus making the antisymmetric mode lower in frequency. Above $\omega_{p}$ a dispersive plane waves travels inside the film that undergoes total reflection at the interfaces. This Figure also depicts the number of extrema for the electric component $E_{z}$, which determines the symmetry of the mode. 
In Fig.(2), the symmetric and antisymmetric dispersion relations are shown for a very thin film $d=10 \mathrm{~nm}$ and the three anisotropies, $\lambda_{\perp} / \lambda_{\|}=5,10^{2}$ and $10^{3}$. In order to slow down as much as possible light in the dielectric, and so, lower the coupled regime frequency range, we choose $\mathrm{SrTiO}_{3}$ as the exterior non-conducting dielectric medium. At low temperatures its dielectric constant is known to be high up to the GHz frequency 18 , $\tilde{\varepsilon} \approx 20000$. All curves in this Figure were directly obtained from the parametrized solution of Eq.(19) and Eq.(20). The symmetric state crossover frequency, $\omega_{\text {cross }, s}$, between the optical and the square root regimes is found to be $75 \mathrm{GHz}$ for the three anisotropies displayed here. The antisymmetric state crossover frequency, $\omega_{\text {cross }, a}$, between the optical and the coupled regime, depends on the anisotropy, being $2.3 \mathrm{THz}, 290 \mathrm{GHz}$ and $48 \mathrm{GHz}$ for $\lambda_{\perp} / \lambda_{\|}=5,10^{2}$ and $10^{3}$, respectively. Because of this anisotropy dependence they are not indicated in this Figure. Notice that the symmetric mode (dashed lines) is always found at a frequency range lower than its corresponding antisymmetric mode (continuous lines). In the low wave vector limit, all the dispersions collapse into the same linear (optical) regime. In the opposite limit, $\beta$ very large, both symmetric and antisymmetric curves converge to the same asymptotic frequency. This asymptotic frequency is just the surface plasma frequency, strongly affected by anisotropy: $1 \mathrm{THz}$ for $Y \mathrm{BCO}, 200 \mathrm{GHz}$ for $\mathrm{BSSCO}$ and $50 \mathrm{GHz}$ for $\lambda_{\perp} / \lambda_{\|}=10^{3}$. This saturation frequency is independent on film thickness, as expected, according to Eq.(29). Notice that the wave vector signaling the onset of the asymptotic regime decreases for increasing anisotropy, ranging from $10 \mu \mathrm{m}^{-1}$ for YBCO to $100 \mathrm{~mm}^{-1}$ for the maximum anisotropy considered here. Between the optical and the asymptotic is the coupled regime, the true plasma mode, found to extend over a large range of frequency and wavenumber for an $Y B C O$ thin film, according to this Figure. In this regime the symmetric plasma mode follows a square root and the antisymmetric mode clearly shows the inverse square root dependence. For $B S C C O$, the coupled regime is shorter than in $Y B C O$, ranging between 0.1 to $5 \mu m^{-1}$. For the maximum anisotropy of $10^{3}$, the coupled regime disappears thus only surviving the optical and the asymptotic regimes. We conclude that an extremely large anisotropy inhibits coupling between the two surfaces and enhances the surface plasma 
modes.

Fig.(3), directly obtained from Eq.(29), shows the asymptotic frequency versus $\tilde{\varepsilon}$, for several anisotropies. Notice that the asymptotic frequency for small $\tilde{\varepsilon}$ is just $\omega_{p}$. For $Y B C O$, the asymptotic frequency drops over an order of magnitude when $\tilde{\varepsilon}$ changes by three decades. The choice of a non-conducting medium of small dielectric constant can render impossible the observation of these plasma modes because the asymptotic frequency becomes larger than the superconducting gap. In $B S C C O$, and other extremely anisotropy compounds, the asymptotic regime is always much smaller than the gap. As $\tilde{\varepsilon}$ increases, we have determined the crossover value, $\varepsilon_{s} \lambda_{\perp} / \lambda_{\|}$, where the assymptotic frequency acquires a $\tilde{\varepsilon}$ dependence, thus being strongly affected by anisotropy. Hence in order that the inverse square-root dependence of the antisymmetric coupled regime be observable, the condition $\tilde{\varepsilon}>\varepsilon_{s} \lambda_{\perp} / \lambda_{\|}$must be satisfied.

Fig.(田) shows the dispersion relations above $\omega_{p}$ for a $100 \mathrm{~nm}$-thick film. The dielectric constant $\tilde{\varepsilon}$ is taken equal to $\varepsilon_{s}$. We have chosen a $B S C C O$ compound with an anisotropy $\lambda_{\perp} / \lambda_{\|}=10^{2}$. Therefore the c-axis plasma frequency is much smaller than the superconducting gap 34. The curves were directly obtained from Eq.(23) and Eq.(24). Above $\omega_{p}$, plasma modes branch into a large number of modes when film thickness increases. For the choice of a 100nm-thick film, the present London-Maxwell theory remains valid for the first 66 modes. We plot in this figure the $M=1,2,3,4,7,12$ and 66 dispersion relations. For increasing $M$ the plasma mode becomes slower, indicating that the number of reflections that the confined plane wave undergoes at the interfaces has also increased. Notice that the $M=66$ plasma mode is very near $\omega_{p}$. The $M=1$ branch is very near to the optical branch showing that it essentially has the speed of light in the dielectric medium.

\section{CONCLUSION}

In this paper we have studied propagating plasma modes in a superconducting film surrounded by two identical dielectric media. The superconductor is anisotropic having its 
uniaxial direction (c-axis) perpendicular to the interfaces with the dielectric medium. We consider the existence of a plasma frequency along the uniaxial direction below the gap and study its effects into the propagating modes using the London-Maxwell theory, which gives a good account of the physical situation for scales larger than the interplane separation. In fact we do use the interplane separation as a cut-off for the present theory. We only consider low incident frequency compare to the superconducting gap frequency. Moreover the wavelength associated to the incident wave is much larger than London's penetration length along the surfaces. Therefore the dielectric constant of the superconducting film along the plane is always negative and with a large modulus. Along the c-axis, the situation is quite distinct. The dielectric constant of the superconducting film along this direction vanishes at the plasma frequency, being positive above. In this paper we were only concerned about modes that propagate parallel to the surfaces and evanesce in the dielectric medium. Under this condition, and the requirement of the non-conducting medium dielectric constant larger than the superconductor's static constant, we find that modes are either totally above the plasma frequency, or below, thus never crossing the plasma frequency line. Thus we study these two regions of frequency separately. Within our London-Maxwell theory framework we find the exact expressions for the dispersion relations of the plasma modes in the two cases. The exact expressions are found in parametric form, the parameter being the one that characterizes the exponential behavior inside the superconductor. Approximated expressions for some especial regimes of the dispersion relations are obtained from our exact parametric solution. In this way we retrieve well-known results in the literature 19272728 to the context of an anisotropic film with a plasma frequency inside, and also, derive new ones. We find that propagating modes below and above the plasma frequency have quite distinct physical properties.

Below the plasma frequency, the amplitude also evanesce inside the superconductor, the film thickness is important in order to assure a sufficiently strong coupling between the surfaces. There are two branches of the dispersion relation corresponding to the two possible arrangements of the superficial charge densities. The symmetric branch is the lowest in 
frequency and has the two superficial charge density symmetrically disposed. The highest branch has opposite charge facing each other at the interfaces thus being antisymmetric. We have studied here in details the three possible regimes that can exist for these two branches, their cross-over, and the conditions for the existence of the coupled regime, the most interesting of the three regimes. In the so-called coupled regime both symmetric and antisymmetric dispersion relation branch provides independent information on the two London penetration lengths. The antisymmetric mode is intimately connected to the transverse current component, thus being highly sensitive to the transverse London penetration length, in the same fashion that the symmetric mode depends on the longitudinal London penetration length. We believe that this remarkable property can be used to gain further understanding of the transverse current component in anisotropic and layered films.

Above the plasma frequency the amplitudes do not evanesce inside the film, propagation is geometrically understood, and an oblique ray can represent the plane wave inside the superconductor which is totally reflected at the interfaces (see Fig.(佃)). However outside the film, in the dielectric medium, the wave remains evanescent, and we conclude that above the plasma frequency the superconducting film displays confined propagation, similar to an optic fiber. Notice that the film thickness is not a crucial parameter because no matter how apart the surfaces are, there will always be plane waves travelling in its interior. Contrary to below the plasma frequency, above there are many modes corresponding essentially to the number of half-wavelengths that fit inside the film along the c-axis.

In summary we have shown in this paper that superconducting films surrounded by a dielectric medium displays very interesting plasma modes propagation properties at frequencies below the gap frequency and such properties are quite distinct below and above the c-axis plasma frequency.

This work was done under a CNRS(France)-CNPq(Brasil) collabotation program. 


\section{APPENDIX}

In this appendix we provide further details on how the exact parametrized solutions of section IV leads the the pictures developed in sections $I I I$ and section IV for below and above the plasma frequency, respectively.

$\underline{\omega<\omega_{p}}$

The three possible regimes, optical coupled and assymptotic follow from the exact parametrized solution. In particular we show that Eq.(25) and Eq.(26), for the coupled regime, and Eq.(29), for the asymptotic regime, follow from the Eq.(19) and Eq.(20). We derive both Eq.(25) and Eq.(26) within the following approximations: (i) retardation effects are neglected $(\gamma \ll 1)\left(\sqrt{1-\gamma^{2}} \approx 1\right)$; (ii) fields evanesce slowly inside the film, $t \ll 1$. Therefore the Eq.(18) dispersion relations become $\sqrt{A X} \gamma^{2} \approx 1 / 2$ and $\sqrt{A X} \gamma^{2} \approx 2 / t^{2}$ for the symmetric and for the antisymmetric modes, respectively; and (iii) the film thickness is much smaller than the London penetration length along the surfaces $A \gg 1$ so that even though $t$ is small we can approximate Eq.(17) by $A t^{2} \approx \sqrt{X R /\left(1-\gamma^{2} X R / r\right)}$. Direct elimination of the parameter $t$ leads to both Eq.(25) and Eq.(26). In the asymptotic regime fields inside the superconductor fade away very quickly from the surfaces, thus corresponding to $t \gg 1$. The surface decoupling is seen from the antisymmetric and symmetric dispersion relations which converge to the same asymptotic frequency $\omega_{a s}$. According to Eq.(19) $g_{A}(t \rightarrow \infty)=g_{S}(t \rightarrow \infty) \rightarrow t^{2}$ and Eq.(29) follows in a straighforward way from this argument.

$\underline{\omega>\omega_{p}}$

To derive Eq.(30) from the exact parametrized solution is in fact very simple, because it does not demand a detailed study of the $\gamma\left(t^{\prime}\right)$ curve. To do so we reparametrize the exact solution of Eq.(23), replacing $t^{\prime}$ by a new parameter $t_{1}, t^{\prime}=t_{I}-t_{1}$ where $t_{S}=(2 N+1) \pi$, and $t_{A}=2(N+1) \pi$. The advantages are two-fold: both symmetric and antisymmetric modes are now defined in the same interval $0 \leq t_{1} \leq \pi$; and the approximation is under 
control, corresponding to the limit $t_{1} / t_{I} \ll 1$. Notice that at $t_{1}=0$ all curves satisfy the condition $\gamma=1$, thus justifying our claim that Eq.(30) is a good approximation for the exact parametrized dispersion relation of Eq.(23) and Eq.(24) when the phase velocity is approximately given by the speed of light in the dielectric. Indeed to obtain Eq.(30) from Eq.(23) and Eq.(24), notice that $\gamma\left(t_{I}-t_{1}\right)$ can be Taylor expanded in powers of $t_{1}$ because the functions $h_{I}\left(t^{\prime}\right)$, introduced in section [IV] are well behaved in this neighborhood: $h_{I}\left(t^{\prime}\right)=\left(t_{I}-t_{1}\right)^{2} \tan ^{2}\left(t_{1} / 2\right)$. We introduce the approximation $t_{1} / t_{I} \ll 1$ into Eq.(24) obtaining that $\left.X_{I} \approx(r / R) /\left\{\gamma_{I}^{2}-r /\left[A\left(t_{I}\right)^{2}+1\right)\right]\right\}$. The key issue here is that all the parametric dependence of $X$ is now on $\gamma$, because the last term was approximated by a constant, $r / A\left(t_{I}-t_{1}\right)^{2} \approx r / A t_{I}^{2}$. Therefore we obtain the approximated parametrized dispersion relation,

$$
\begin{aligned}
& \omega\left(t_{1}\right) \approx \omega_{p} \sqrt{\frac{\gamma_{I}\left(t_{1}\right)^{2}}{\gamma_{I}\left(t_{1}\right)^{2}-\frac{r}{A t_{I}^{2}}}} \\
& \beta\left(t_{1}\right) \approx \beta_{c} \sqrt{\frac{1}{\gamma_{I}\left(t_{1}\right)^{2}-\frac{r}{A t_{I}^{2}}}}
\end{aligned}
$$

from where the family of curves $\omega(\beta)$ of Eq.(30) follow, by suitably removing the function $\gamma(t 1)$.

Finally we would like to get some more detailed information about the parametrized exact solution, in particular the two following issues: $(i) \omega(\beta)$ is always an increasing function of $\beta$; (ii) near $\omega_{p}$ it suffices to consider the positive root of Eq.(23) because the positive and negative roots of Eq.(23) are just parts of the same curve and meet where the square root vanishes. The claims are of general validity but we derive them under some further working assumptions on Eq.(23), that in leading order, acquires a very simple form,

$$
A\left(t_{I}-t_{1}\right)^{2} \gg r \gg 1, \quad \gamma_{I}\left(t_{1}\right)^{2}=\frac{1 \pm \sqrt{1-4 \frac{r^{2}}{R} \tan ^{2}\left(\frac{t_{1}}{2}\right)}}{2\left[1+\frac{A r}{R}\left(t_{I}-t_{1}\right)^{2} \tan ^{2}\left(\frac{t_{1}}{2}\right)\right]}
$$

To have the condition $r \gg 1$ satisfied one must choose a dielectric medium of sufficiently high constant: $\tilde{\varepsilon} \gg \varepsilon_{s}$. To have at least one mode described by the above inequality, one must require that the minimal frequency satisfies the inequality: $t_{\max }^{\prime} \gg \sqrt{r / A}$, which is 
$\sqrt{\tilde{\varepsilon} / \varepsilon_{s}} \ll 2 \pi \lambda_{\|} / a$. To show that the curve never saturates, take $t_{1}=0$, where one finds that $\gamma=1$ and $\gamma=0$ for the positive and negative roots, respectively. Obviously the positive root is the low frequency part of the curve. Notice that the negative root is not physical because for $\gamma=0 \beta(X)$ is negative according to Eq.(24). This just means that the negative square root curve must end at a finite non-zero $\gamma$, where the denominator of Eq.(24) vanishes and so $X$ diverges. $\gamma$ is limited between zero and one, so it must also be finite at this point. Consequently $\omega$ must also diverge, and so, we conclude from this that the curve never saturates in $\omega$ or $\beta$. 


\section{REFERENCES}

${ }^{1}$ K. Tamasaku, Y. Nakamura, and U. Uchida, Phys. Rev. Lett. 69, 1455 (1992).

${ }^{2}$ A.M. Gerrits, A. Wittlin, V.H.M. Duijn, A.A. Menovsky, J.J.M. Franse, and P.J.M. van Bentum, Physica C 235-240, 1117 (1994).

${ }^{3}$ J.H. Kim, H.S. Somal, M.T. Czyzyk, D. van der Marel, A. Wittlin, A.M. Gerrits, V.H.M. Duijn, N.T. Hien, and A.A. Menovsky, Physica C 247, 297 (1995).

${ }^{4}$ C.C. Homes, T. Timusk, R. Liang, D.A. Bonn, and W.N. Hardy, Phys. Rev. Lett. 71, 1645 (1993).

${ }^{5}$ S. Tajima, G.D. Gu, S. Miyamoto, A. Odagawa, and N. Koshizuka, Phys. Rev. B 48, 16164 (1993).

${ }^{6}$ Ophelia K.C. Tsui, N.P. Ong, Y. Matsuda, Y.F. Yan, and J.B. Peterson, Phys. Rev. Lett., 73, 724 (1994).

${ }^{7}$ Ophelia K.C. Tsui, N.P. Ong, and J.B. Peterson, Phys. Rev. Lett., 76, 819 (1995).

${ }^{8}$ Y. Matsuda, M.B. Gaifullin, K. Kumagai, K. Kadowaki, and T. Mochiku, Phys. Rev. Lett., 75, 4512 (1995).

${ }^{9}$ P.W. Anderson, Phys. Rev. 112, 1900 (1958).

10 T. Mishonov, Phys. Rev. B 44, 12033 (1991); 50, 4004 (1994).

${ }^{11}$ S.N. Artemenko and A.G. Kobelkov, JETP Lett. 58, 445 (1993);

${ }^{12}$ H.A. Fertig and S. Das Sarma, Phys. Rev. Lett. 65, 1482 (1990), and Phys. Rev. B 44, $4480(1991)$.

${ }^{13}$ Y.B. Kim and X.G. Wen, Phys. Rev. B 48, 6319 (1993).

${ }^{14}$ L.N. Bulaevskii, M. Zamora, D. Baeriswyl, H. Beck, and J.R. Clem, Phys. Rev. B 50, 12831 (1994). 
15 J.E. Mooij and G. Schön, Phys. Rev. Lett. 55, 114 (1985).

${ }^{16}$ B. Mirhashem and R. Ferrell, Physica C 161, 354 (1989).

17 T. Mishonov and A. Groshev, Phys. Rev. Lett. 64, 2199 (1990).

${ }^{18}$ O. Buisson, P. Xavier, and J. Richard, Phys. Rev. Lett. 73, 3153 (1994); Phys. Rev. Lett. 74E, 1493 (1995).

${ }^{19}$ F.J. Dunmore, D.Z. Liu, H.D. Drew, and S. Das Sarma, Phys. Rev. B 52, R731 (1995).

${ }^{20}$ H. Boersch, J. Geiger, A. Imbush, and N. Niedrig, Phys. Lett. 22, 146 (1966).

${ }^{21}$ M. Fukui, V.C.Y. So, and R. Normandin, Phys. Stat. Sol. 91, K61 (1979).

${ }^{22}$ D. Sarid, Phys. Rev. Lett. 47, 1927 (1981).

${ }^{23}$ S. Ushioda and R. Loudon, in Surface Polaritons, edited by V.M. Agranovich and A.A. Maradudin (North-Holland P.Co., New York, 1982), p.573.

${ }^{24}$ E.N. Economou, Phys. Rev. 182, 539 (1969).

${ }^{25}$ K.L. Kliewer and R. Fuchs, Phys. Rev. 153, 498 (1966).

${ }^{26}$ P.K. Tien and R. Ulrich, J. Opt. Soc. Amer. 60, 1325 (1970).

${ }^{27}$ M.M. Doria, F. Parage, and O. Buisson, Europhys. Lett. 35, 445 (1996).

28 S.N. Artemenko and A.G. Kobelkov, Physica C 253, 373 (1995).

${ }^{29}$ P. Yeh, Optical Waves in Layered Media (John Wiley \& Sons, New York, NY, 1988).

${ }^{30}$ O. Buisson, F. Parage, and J. Richard in Macroscopic Quantum Phenomena and Coherence in Superconducting Networks ed. C. Giovannella, M. Tinhkam (World Scientific, 1995) p25.

${ }^{31}$ G. Blatter et al., Rev. Mod. Phys. 66, 1125 (1994).

${ }^{32}$ K.E. Gray, R.T. Kampwirth, and D.E. Farrel, Phys. Rev. B 41, 819 (1990). 
${ }^{33}$ E.H. Brandt, Rep. Prog. Phys. 58, 1465 (1995).

34 Tunneling and proximity effect studies give the frequency gaps $5.010^{12} \mathrm{~Hz}$, and $7.510^{12} \mathrm{~Hz}$, for $Y B C O$ and $B S C C O$, respectively. See, for instance, M.R. Beasley, Physica C 185, 227 (1991). 


\section{FIGURES}

FIG. 1. A pictorial view of wave propagation in a superconducting film surrounded by two equivalent non-conducting media. For $\omega<\omega_{p}$ the instantaneous electric field and the superficial charges are shown for symmetric (a) and antisymmetric (b) modes. For $\omega>\omega_{p}$ the optical ray associated to the plane wave travelling inside the film is shown here (c) for $M=1,2,3$ and 4 . The symmetry of each state is also shown here, for both cases (a), (b) and (c), through the diagram $E_{z}$ versus $x$.

FIG. 2. The symmetric and antisymmetric $\omega<\omega_{p}$ dispersion relations are shown for three anisotropies. A very thin superconducting film, $d=10 \mathrm{~nm}$-thick, surrounded by $\mathrm{SrTiO}_{3}$, is considered.

FIG. 3. This Figure shows the asymptotic frequency versus $\tilde{\varepsilon}$, for three anisotropies. For small $\tilde{\varepsilon}$ the asymptotic frequency is just $\omega_{p}$. The asymptotic frequency for $Y B C O$ is the most sensitive to changes in the susbstrate dielectric constant.

FIG. 4. The dispersion relations above $\omega_{p}$ is shown for a $100 \mathrm{~nm}$-thick film and anisotropy $\lambda_{\perp} / \lambda_{\|}=100$. The $M=1$ mode propagates with speed $v$ and at the $M=66$ mode is the upper limit for the validity of the present theory. 


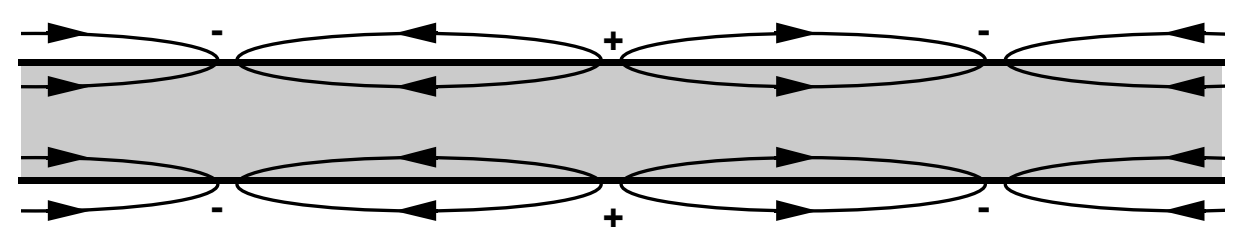

(a)

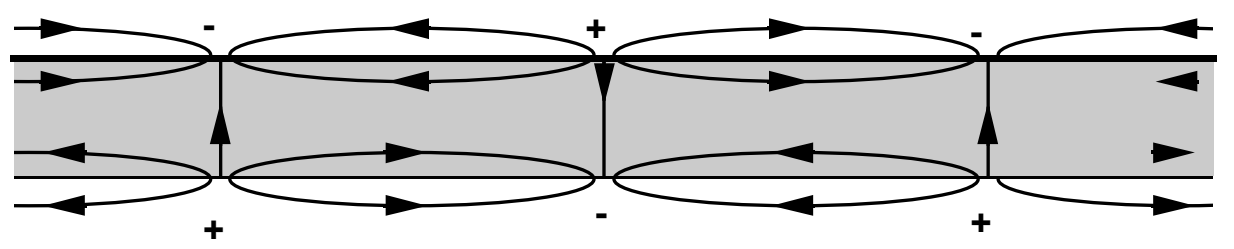

(b)
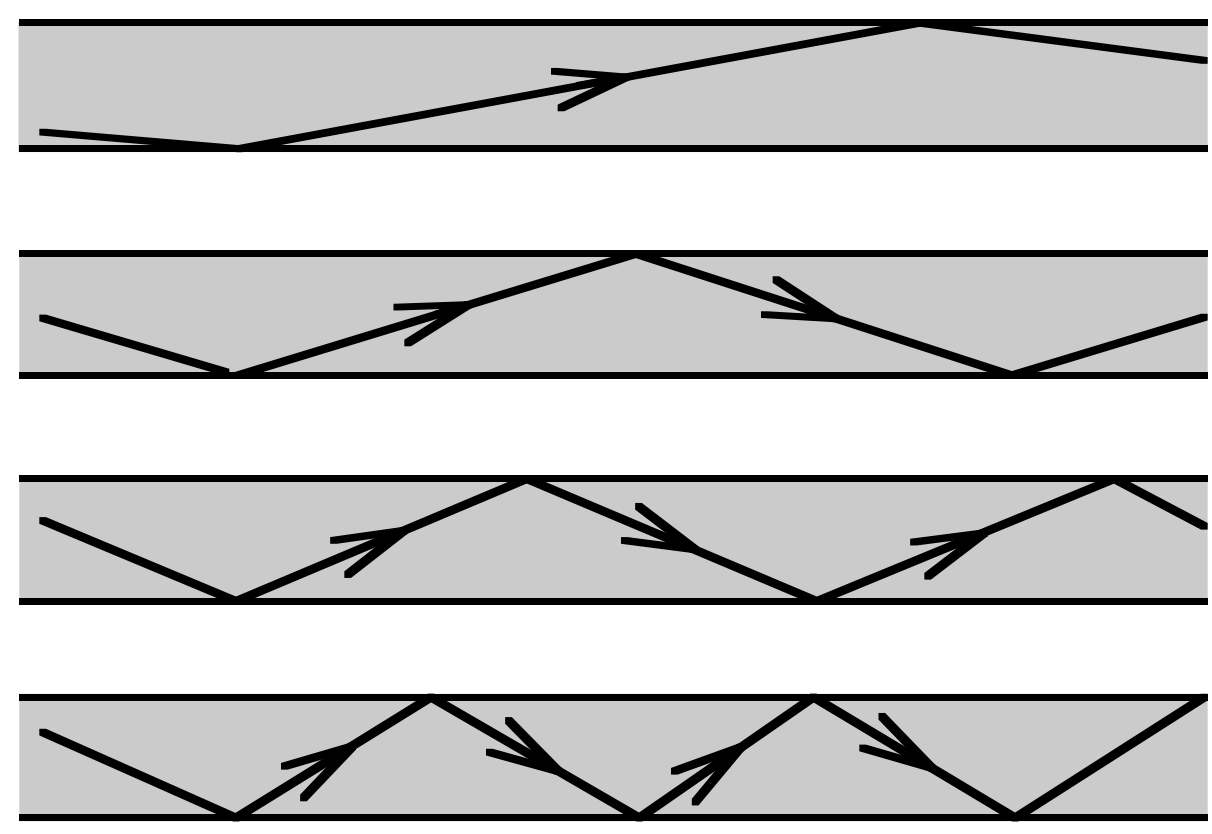

(c)
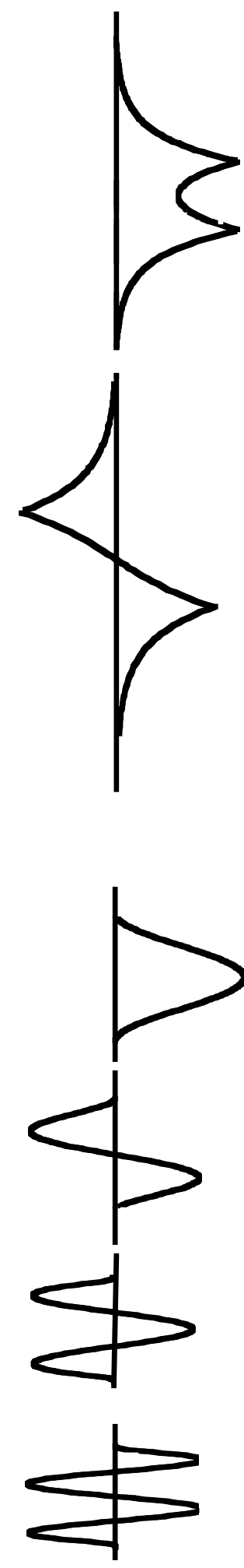


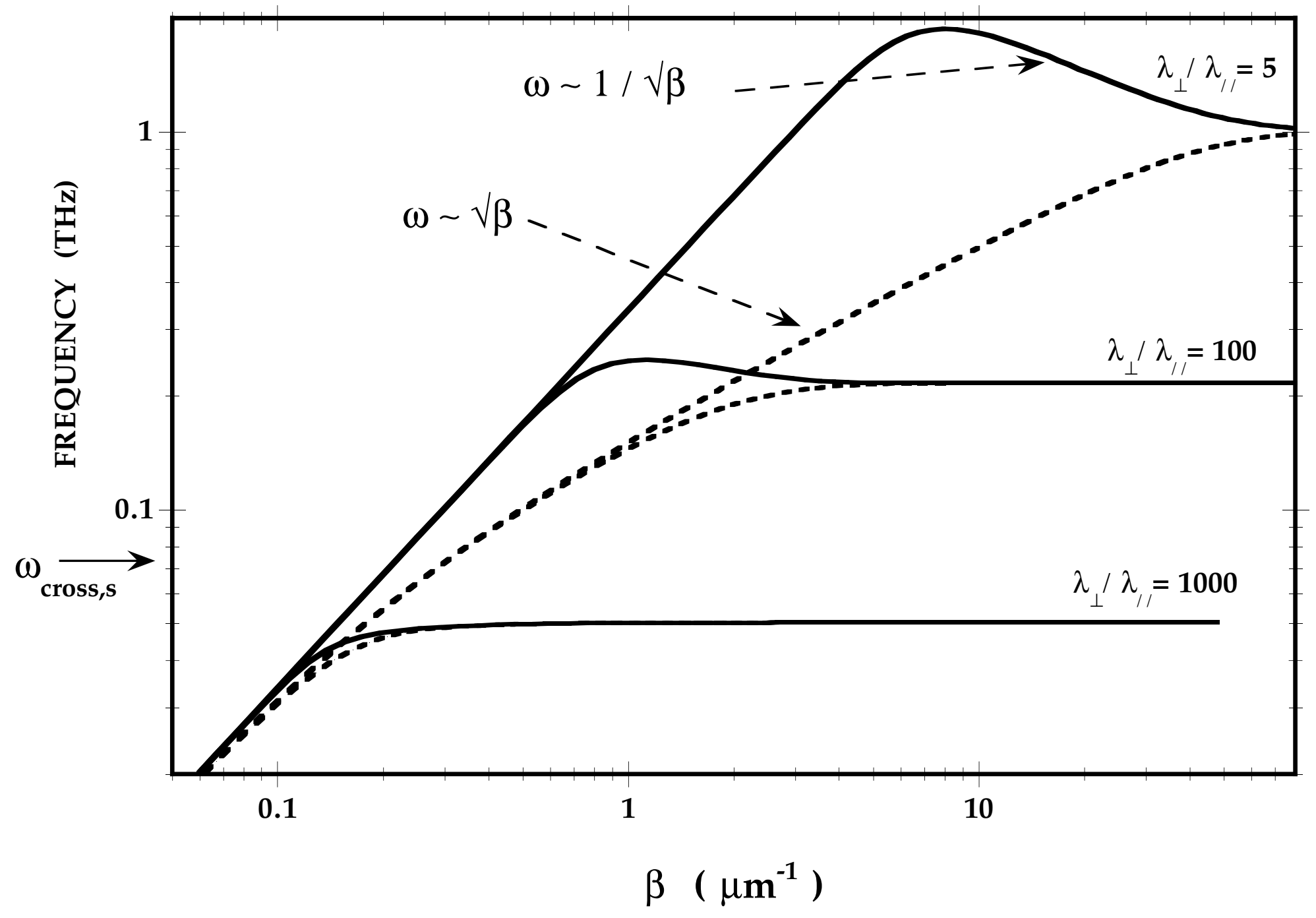




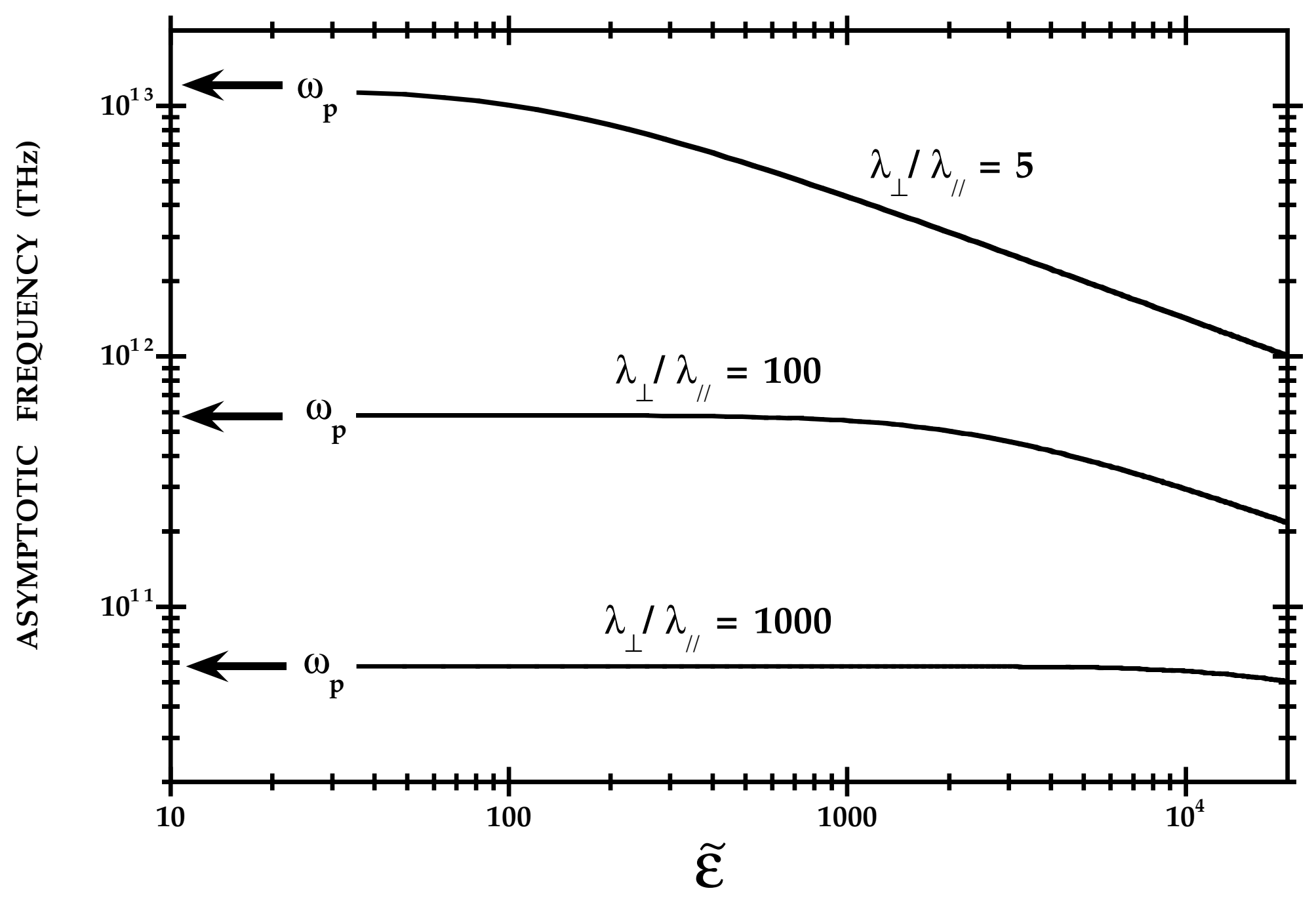




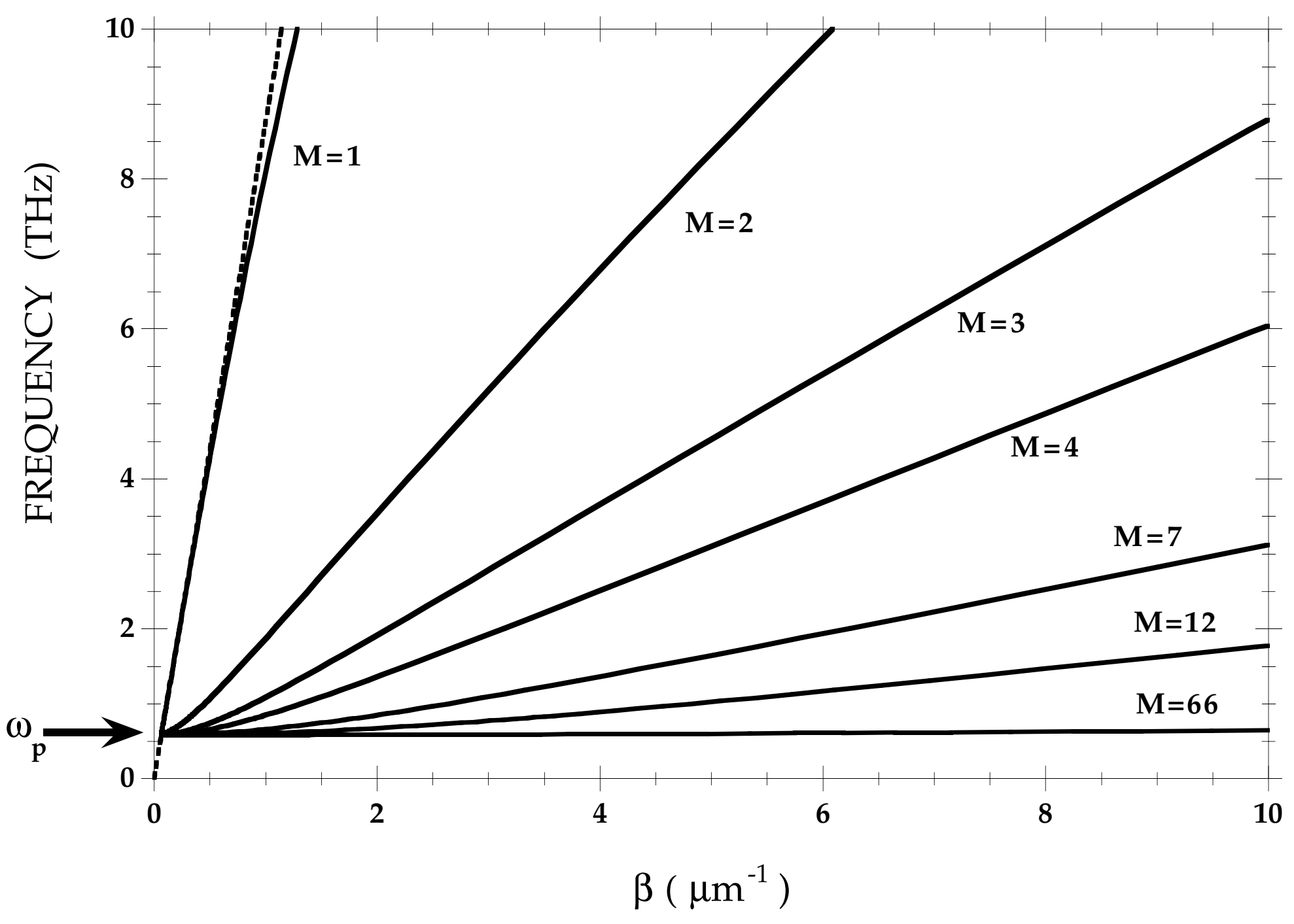

